\title{
STOCHASTIC SCHEDULING WITH MULTIPLE RESOURCE CONSTRAINTS USING A SIMULATED ANNEALING-BASED ALGORITHM
}

\author{
Po-Han Chen \\ Assistant Professor \\ School of Civil \& Environmental Engineering \\ Nanyang Technological University, Singapore \\ cphchen@ntu.edu.sg
}

\author{
Seyed Mohsen Shahandashti \\ Mater of Engineering Student \\ School of Civil \& Environmental Engineering \\ Nanyang Technological University, Singapore \\ seye0001@ntu.edu.sg
}

\begin{abstract}
This paper proposes a new algorithm using Simulated Annealing (SA) for stochastic scheduling. Stochastic effects are added with nondeterministic activity durations. Simple simulated annealing can not properly handle such a complex problem so an improved version is produced and utilized within the algorithm. Generally, the availability of resources is not enough to complete all the current activities of the project and activities compete for multiple resources which stimulates considering multiple resource constraints in formulation. The superiority of the model over one of the famous heuristics is verified by a numerical example using Monte Carlo method (MC).
\end{abstract}

\section{KEYWORDS}

Stochastic scheduling, simulated annealing, Monte Carlo method, multiple resource constraints

\section{INTRODUCTION}

Effective project scheduling has been one of the main challenges in project planning since Critical Path Method (CPM) and Program Evaluation and Review technique (PERT) have been developed. $\mathrm{CPM}$ and PERT handle projects with deterministic and stochastic durations respectively.

Although lots of research has been done in the area of deterministic scheduling to find the optimal start times of activities to minimize the project duration when there are limited resources, there are few techniques to handle stochastic scheduling [1].
Monte Carlo simulation is utilized in this paper to produce the average and variance of distribution function of project duration considering resourceconstrained scheduling method. First it is needed to produce deterministic sample values for stochastic values of activity durations from respected distributions in each realization. It is possible to build a close-to-true distribution function for project duration using Monte Carlo simulation with enough realization number [2]. So it is feasible to utilize this method to produce distributed functions for the schedule built by our algorithm and other algorithms and compare these distribution functions in order to 
show the usefulness of the algorithm proposed in this paper.

A simple simulated annealing consists of several decreasing temperatures and it is based on the similarity between the solid annealing process and solving the combinatorial optimization problem [3]. Each temperature includes a sequence of iterations. First, the beginning temperature is chosen and the initial solution is selected and the value of the cost function based on the current solution will be calculated; then a new solution will be created in the neighborhood of the previous solution. The new value of the cost function will be calculated. If the new value of the cost function is less than the previous one, it will be accepted. If the new value of the cost function is more than the previous one, it will be accepted according to Metropolis's criterion based on Boltzman's probability [4]. According to Metropolis's criterion, if the difference between the cost function values of the current and the newly produced solutions $(\Delta \mathrm{E})$ is equal to or larger than zero, a random number $\delta$ in $[0,1]$ is generated from a uniform distribution and if

$$
\delta \leq e^{(-\Delta E / T)}
$$

then the newly produced solution is accepted as the current solution. The number of new solutions created in each temperature is as many as the iteration number which is limited by the termination condition. The termination condition can simply be a certain number of moves. Then temperature will reduce upon a temperature update rule and every above-said step will iterate until the algorithm reaches the halting criteria. The halting criteria can be the status in which the temperature goes down the minimum temperature. The temperature update rule used in this research is shown in equation 2 .

$$
\text { Temperature }=T e^{(-r t)}
$$

where $\mathrm{T}$ is the initial temperature, $\mathrm{r}$ is the cooling ratio, and $t$ is the number of times that temperature has been decreased.

\section{PROBLEM DEFINITION}

Resource availabilities are not sufficient for all the current activities to be started. (Current activities are those whose predecessor activities are completed) So only some of them have the chance to be accepted. This can be modeled by a series of priority rules showing the priority of activities over one another. In the other words, current activities with higher priority can be started. In order to do this, we assign each activity a number between zero and $\mathrm{N}$ including zero. This number is called Priority Index (PI). There are not two activities with the same PI. The objective is to find the best set of Priority Indices for activities to achieve the following goal:

$$
\begin{aligned}
& \text { Minimize } A V E^{m} \times \operatorname{Var}^{n} \\
& A V E=\frac{\sum_{i=1}^{R}\left[\max \left\{f\left(n, X_{i, n}\right) \mid n=1, \ldots, N\right\}\right]}{R} \\
& V A R=\frac{\sum_{i=1}^{R}\left[\max \left\{f\left(n, X_{i, n}\right) \mid n=1, \ldots, N\right\}-A V E\right]^{2}}{R}
\end{aligned}
$$

The objective function is minimization of multiplication of two parts. The first part is the average of project durations in $\mathrm{R}$ realizations. The second part is the variance of project durations in $\mathrm{R}$ realizations. $\mathrm{m}$ and $\mathrm{n}$ are powers of the first and second parts showing the importance of each of the parts for the scheduler. A project manager should run the program with a variety of $m$ and $n$ values to find the best combination for his project based on his experience and the nature of his project. For instance, probably a project manager prefers to reduce the scheduling risk. In this case, he will probably increase $n$. On the other hand, another one may prefer to increase the chance of decreasing the average even if the risk is higher. In this case, he will increase $\mathrm{m}$.

The precedence relationships are the problem constraint which can be defined as follows:

$$
s\left(n, X_{i, n}\right) \geq f\left(p, X_{i, n}\right) \mid \forall i=1, . ., R ; \forall p \in P(F S)
$$

Stochastic activity durations are modeled by Beta distribution.

$$
X \sim \operatorname{Beta}\left(\alpha_{n}, \beta_{n}\right)
$$


The parameters of Beta distribution can be obtained by the following formulas ([5], [6]):

$\phi_{n}=\frac{5 a_{n}-4 m_{n}-b_{n}}{a_{n}+4 m_{n}-5 b_{n}}$

$\alpha_{n}=\phi_{n} \times \beta_{n}$

$\beta_{n}=\frac{-\left(\phi_{n}^{2}-34 \phi_{n}+1\right)}{\left(\phi_{n}+1\right)^{3}}$

The resource constraints are added to the model as the following:

$r e s_{j, t} \leq R E S_{j, t} \mid \forall j=1, \ldots ., J ; \forall t=1, \ldots T$

Notation:

$\mathrm{N}$ : number of activities

$\mathrm{R}$ : number of realizations

$X_{i, n}$ : the ith generated random variable for $n$th activity

$f\left(n, X_{i, n}\right)$ : the finish time of nth activity when its duration is a random variable

$s\left(n, X_{i, n}\right):$ the start time of nth activity when its duration is a random variable

$a_{n}$ : optimistic time estimate of nth activity

$b_{n}:$ pessimistic time estimate of nth activity

$m_{n}$ : most likely time estimate of nth activity

$r e s_{j, t}$ : the assigned number of resource $\mathrm{j}$ at time $\mathrm{t}$

$R E S_{j, t}$ : the available number of resource $\mathrm{j}$ at time $\mathrm{t}$

$\mathrm{m}$ : the importance factor of average reduction

$\mathrm{n}$ : the importance factor of variance reduction

$\mathrm{P}(\mathrm{FS})$ : set of precedence activities of a certain activity

\section{SOLUTION METHODOLOGY}

The simulated Annealing-based algorithm which is designed to find the best set of priority indices to reach the objective is depicted in Figure 1.

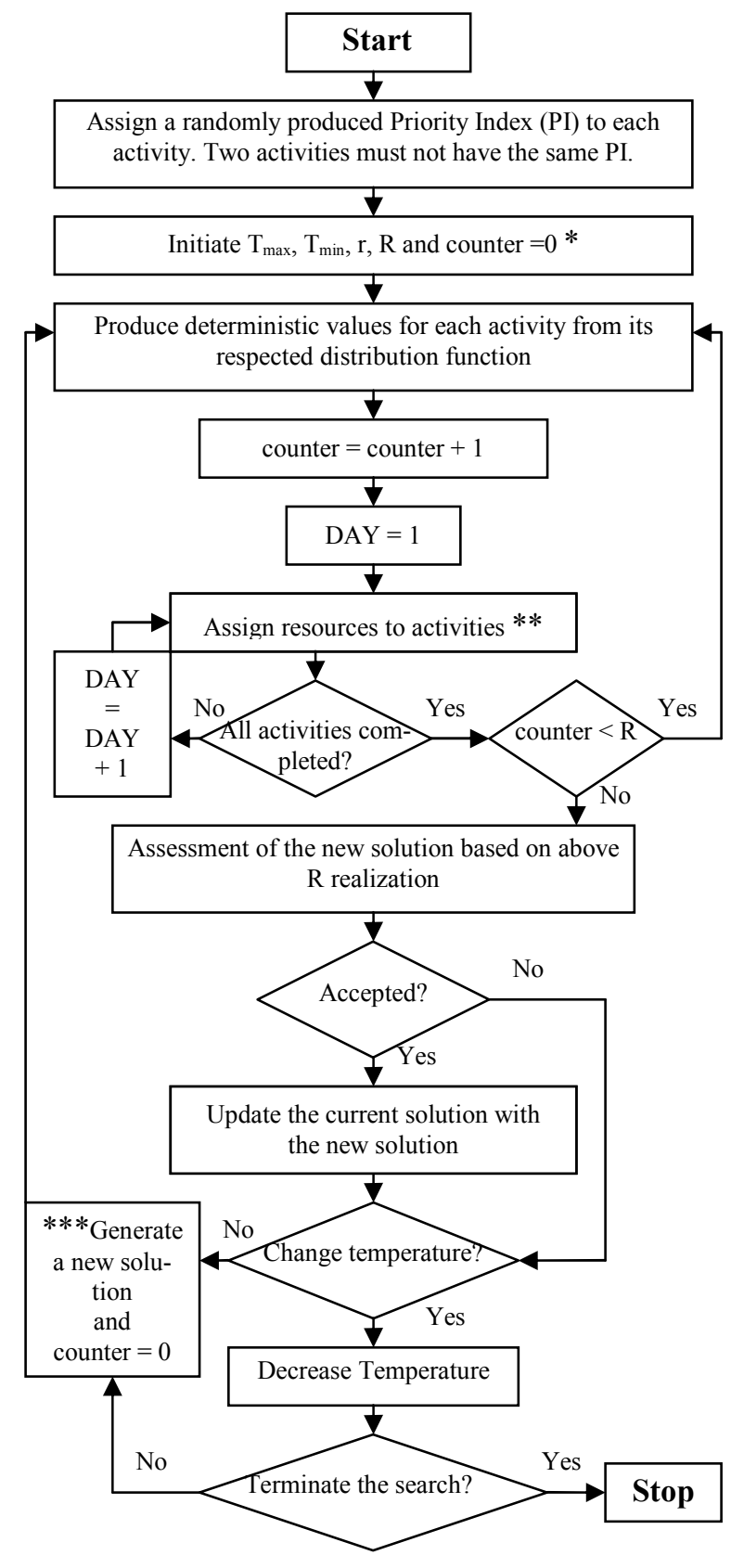

Figure 1. Proposed Algorithm for stochastic scheduling 
* $\mathrm{R}$ represents the number of realizations in each iteration. The nature of the problem is stochastic so it is not logical to use one realization in order to compare solutions of consecutive iterations. So an objective function consisting of the combination of the average and variance of $R$ realizations is produced.

**Resources should be assigned to the current activities. If available resources are not sufficient to handle all the current activities, the priority is given to activities with higher priority indices.

$* * *$ In order to generate a new solution, two priority indices of two activities are swapped. This method produces a neighbor solution for the current solution.

\section{NUMERICAL EXAMPLE}

This section exemplifies the algorithm using a numerical example which is built based on rules proposed by Kolisch and Sprecher [7]. Our example has 50 nodes and 87 activities and the network complexity factor is set to 1.8 . There are 4 resource kinds which all activities compete for. The daily availabilities of resources are randomly produced in the range of. The resource consumption of each activity is uniformly distributed in the range of $[1,4]$. Beta distribution is used to represent random durations of activities. The optimistic, pessimistic and most likely time estimates of each activity are random variables in the range of [1]. The numbers of preceding and successor nodes of each activity are randomly produced in the range of $[1,3]$. The number of start and finish activities are set to 1 .

Maximum and Minimum temperatures are fixed to 1000 and 1 respectively. The cooling ratio is 0.1 and the number of iterations in each temperature is 100 . $\mathrm{R}$ (the number of realization in each iteration) is set to 10 .

Java 5.0 is used as a programming language to encode and compile the implementations, which are run on a personal computer with a Centrino Duo $1.83 \mathrm{GHz}$ CPU and $1 \mathrm{~GB}$ RAM.

In order to show the usefulness of the proposed algorithm and its superiority over other methods, Monte Carlo method has been used. 10000 samples of project durations have been produced for each of SAbased algorithm and FCFS (First Come First Served) algorithm. The results are presented in Table 1.
Table 1. Average, Variance, the multiplication of variance and average and run time of project durations in 10000 realizations produced by the proposed method and FCFS algorithm (Comparison by Monte Carlo Simulation)

\begin{tabular}{|c|c|c|c|c|}
\hline Algorithm & Ave & Var & AvexVar & $\begin{array}{c}\text { Run } \\
\text { Time }\end{array}$ \\
\hline $\begin{array}{c}\text { FCFS } \\
\text { First } \\
\text { Come } \\
\text { First } \\
\text { Served) }\end{array}$ & 177.3 & 17.09 & 3030.06 & $\begin{array}{c}15 \mathrm{~min} \\
30 \mathrm{sec}\end{array}$ \\
\hline $\begin{array}{c}\text { SA-based } \\
\text { Algorithm } \\
\text { m=3,n=1 }\end{array}$ & 180.7 & 0.85 & 153.6 & $\begin{array}{c}58 \mathrm{~min} \\
59 \mathrm{sec}\end{array}$ \\
\hline $\begin{array}{c}\text { SA-based } \\
\text { Algorithm } \\
\text { m=2,n=1 }\end{array}$ & 181.4 & 0.52 & 94.33 & $\begin{array}{c}58 \mathrm{~min} \\
59 \mathrm{sec}\end{array}$ \\
\hline $\begin{array}{c}\text { SA-based } \\
\text { Algorithm } \\
\text { m=1,n=1 }\end{array}$ & 186.4 & 0.47 & 87.61 & $\begin{array}{c}58 \mathrm{~min} \\
59 \mathrm{sec}\end{array}$ \\
\hline $\begin{array}{c}\text { SA-based } \\
\text { Algorithm } \\
\text { m=1,n=2 }\end{array}$ & 195.3 & 0.37 & 72.26 & $\begin{array}{c}58 \mathrm{~min} \\
59 \mathrm{sec}\end{array}$ \\
\hline $\begin{array}{c}\text { SA-based } \\
\text { Algorithm } \\
\text { m=1,n=3 }\end{array}$ & 187.3 & 0.37 & 69.30 & $\begin{array}{c}58 \mathrm{~min} \\
59 \mathrm{sec}\end{array}$ \\
\hline
\end{tabular}

\section{CONCLUSION}

This paper proposes an algorithm for stochastic scheduling of projects with multiple resource constraints. A numerical example verifies the model. Monte Carlo simulation is used as a benchmark method to show the superiority of the SA-based algorithm.

Based on the results shown in Table 1, the proposed model is highly effective in terms of reducing the multiplication of average and variance. In the other words, the proposed model produces schedules which works better considering stochastic effects. The produced schedule gives low project durations with more certainty in random realizations. 
One of the other advantages of the proposed model is the flexibility being incorporated by $\mathrm{m}$ and $\mathrm{n}$ parameters. It makes the model applicable for various kinds of projects and different management styles.

Although the run time of the proposed model is more than that of FCFS, it is reasonable and applicable for real projects.

Multiple resource constraints are incorporated in the formulation and algorithm enabling the model to handle scheduling of real projects.

\section{REFERENCES}

[1] Pet-Edwards, J.\& Mollaghasemi, M. (1996) A Simulation and Genetic Algorithm Approach to Stochastic Research Constrained Project Scheduling, Southcon/96. Conference Record, 25-27 June 1996, Orlando, FL, USA.

[2] Yao, M. J., Chu, W. M. (2007) "A New Approximation Algorithm for Obtaining the Probability Distribution Function for Project Completion Time", Computers and Mathematics with Applications, Vol. 54, pp. 282-295.

[3] Kirkpatrick, S., Gelatt, C. D. Jr and Vecchi, M. P. (1983) "Optimization by Simulated Annealing", Science, Vol. 220, No. 4598, pp. 671-680.

[4] Metropolis, N., Rosenbluth, A. W., Rosenbluth, M. N., Teller, A. H. and Teller, E. (1953) "Equation of State Calculations by Fast Computing Machines", Journal of Chem. Phys., Vol. 21, No. 6, pp. 10871092.

[5] Badiru, A. B. (1991) "A Simulation Approach to Pert Network Analysis", Simulation 57, 245-255.

[6] Baridu, A. B. and Pulat, P. S. (1995) "Comprehensive Project Management", Prentice-Hall, Englewood Cliffs, NJ.

[7] Kolisch, R. and Sprecher, A. (1996) "PSPLIB - A Project Scheduling Library", European Journal Of Operations Research, Vol. 96, 205-216. 